\title{
MILLIMETER OBSERVATIONS OF MARKARIAN GALAXIES
}

\author{
Endrik Krügel \\ Max-Planck-Institute for Radioastronomy \\ Auf dem Hügel 69, 5300 Bonn, F.R.G.
}

\begin{abstract}
We report published data on dust emission from Markarian galaxies and discuss them together with recent spectroscopic work.
\end{abstract}

The following account is brief and avoids repeating information that is already in the literature. Recently, 23 Markarian galaxies have been measured in the continuum at $1.3 \mathrm{~mm}$ with an 11 " beam [1,2]. The sample consists mainly of seyfert and star burst galaxies. The spectral index between 0.1 and $1.3 \mathrm{~mm}$ points to dust emission and the $1.3 \mathrm{~mm}$ fluxes can therefore be converted into gas masses, Mg. Assuming that the total luminosity $\mathrm{L}$, which comes to a large part from the IRAS bands, is also contained in an area $11^{\prime \prime}$ across, one finds the significant relation $\mathrm{L} \alpha \mathrm{M}_{\mathrm{g}}{ }^{0.74}$.

A similar relation $L \alpha \mathrm{M}_{\mathrm{g}}{ }^{0.9}$ has been found in a $90^{\prime \prime}$ beam for bright dusty IRAS galaxies, but with $L / M_{g}$ a factor of 20 lower [3]. It was argued in $[1,2]$ that in both samples the luminosity is derived from star formation. The bright IRAS galaxies are considered to be inactive and therefore have a much lower $\mathrm{L} / \mathrm{M}_{\mathrm{g}}$ ratio than the active Markarians. The clear distinction in the $\left(L, M_{\mathrm{q}}\right)$-plane between the Markarians and the bright IRAS galaxies is probably not a mere beam size effect that would arise if the luminosity came from a point source and if the gas were extended. In this case one would expect, first, a much larger intrinsic scatter in the relation L vs. $M_{g}$ for both samples. Second, the mean linear size of the beam on the galaxy is only twice as large for the bright IRAS galaxies than for the Markarians. Third, objects of a sample of radio-quiet quasars, which are definitely point sources, lie in the $\left(L, M_{g}\right)$-plane in the extension of the Markarians [4].

Although merging may frequently cause the activity in Seyfert and star burst galaxies, one should not draw the conclusion that any other mechanism is excluded. A considerable fraction of the Markarians in our sample do not show any signs of interaction with another galaxy [5]. How an isolated galactic nucleus can undergo repetetive bursts of star formation has been worked out in detail [6]; this scenario readily explains the basic features of the above mentioned observations concerning the Markarians and the bright IRAS galaxies.

The $1.3 \mathrm{~mm}$ continuum measurement of Arp220 with a flux of $1.8 \pm 0.5$ Jy in a 90" beam as reported in [3] for the bright IRAS galaxies may be erroneous. An 11" resolution map at $1.3 \mathrm{~mm}$ from Pico Veleta gives only a flux of $370 \mathrm{mJy}$ in the central $30^{\prime \prime}$ by $30^{\prime \prime}$ region and no detectable flux 373

D. E. Osterbrock and J. S. Miller (eds.), Active Galactic Nuclei, 373-375.

(C) 1989 by the IAU. 
over the rest of the galaxy [7]. This is in agreement with the results quoted for this source during the conference [8]. Arp220 therefore should be placed in the activity strip in Fig.3 of paper [1]. However, even with this reduced value one derives a gas mass of $1.110^{11} \mathrm{M}_{\odot}$. This is still almost an order of magnitude larger than the gas mass of $1.310^{10} \mathrm{M}_{\odot}$ obtained from the $\mathrm{CO}(1-0)$ luminosity in a 45"beam [9].

With the aim of comparing gas masses derived from $\mathrm{CO}, \mathrm{M}_{\mathrm{g}}(\mathrm{CO})$, with those derived from the dust continuum, $M_{g}$ (dust), we observed half of our sample of Markarians in $\mathrm{CO}(1-0)$ and $(2-1)$ with beams of $23^{\prime \prime}$ and $11 "$, respectively [10]. $\mathrm{M}_{\mathrm{g}}(\mathrm{CO})$ was determined from $(2-1)$ in exactly the same manner as from $(1-0)$. We find $\log \left[\mathrm{M}_{\mathrm{g}}\right.$ (dust) $\left./ \mathrm{M}_{\mathrm{g}}(\mathrm{CO} 2-1)\right]=-0.05 \pm 0.38$, which refers to identical beams, and $\log \left[M_{g}(\right.$ dust $\left.) / M_{g}\left(\begin{array}{ll}C O & 1-0\end{array}\right)\right]=$ $-0.45 \pm 0.34$. For higher masses the ratio $M_{g}(d u s t) / M_{g}(C O)$ tends to increase for both lines. There are two kinds of spectra: those that can be fit with one Gaussian and others that consist of two components. The latter class prevails. A few spectra are shown for illustration.

\section{REFERENCES}

[1] E. Krügel, R. Chini, E. Kreysa, W. Sherwood: 1988, A\&A 190, 47

[2] E. Krügel, R. Chini, E. Kreysa, W. Sherwood: 1988, A\&A 193, L16

[3] R. Chini, E. Kreysa, E. Krügel, P.G. Mezger: 1986, A\&A 166, L10

[4] R. Chini, E. Kreysa, P. Biermann: 1988, A\&A submitted

[5] J. Mazarella, V. Balzano: 1986, ApJ Suppl. 62, 751

[6] H. Loose, E. Krügel, A. Tutukov: 1982, A\&A 105, 342

[7] R. Zylka, priv. communication

[8] J. Keene, this conference

[9] D. Sanders, I. Mirabel: 1985, ApJ 298, L31

[10] R. Chini, E. Krügel, H. Steppe: in preparation

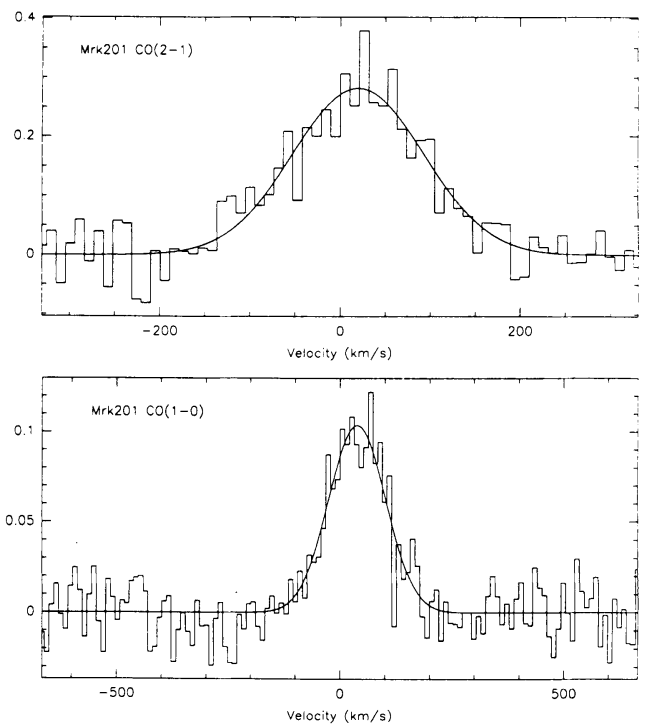



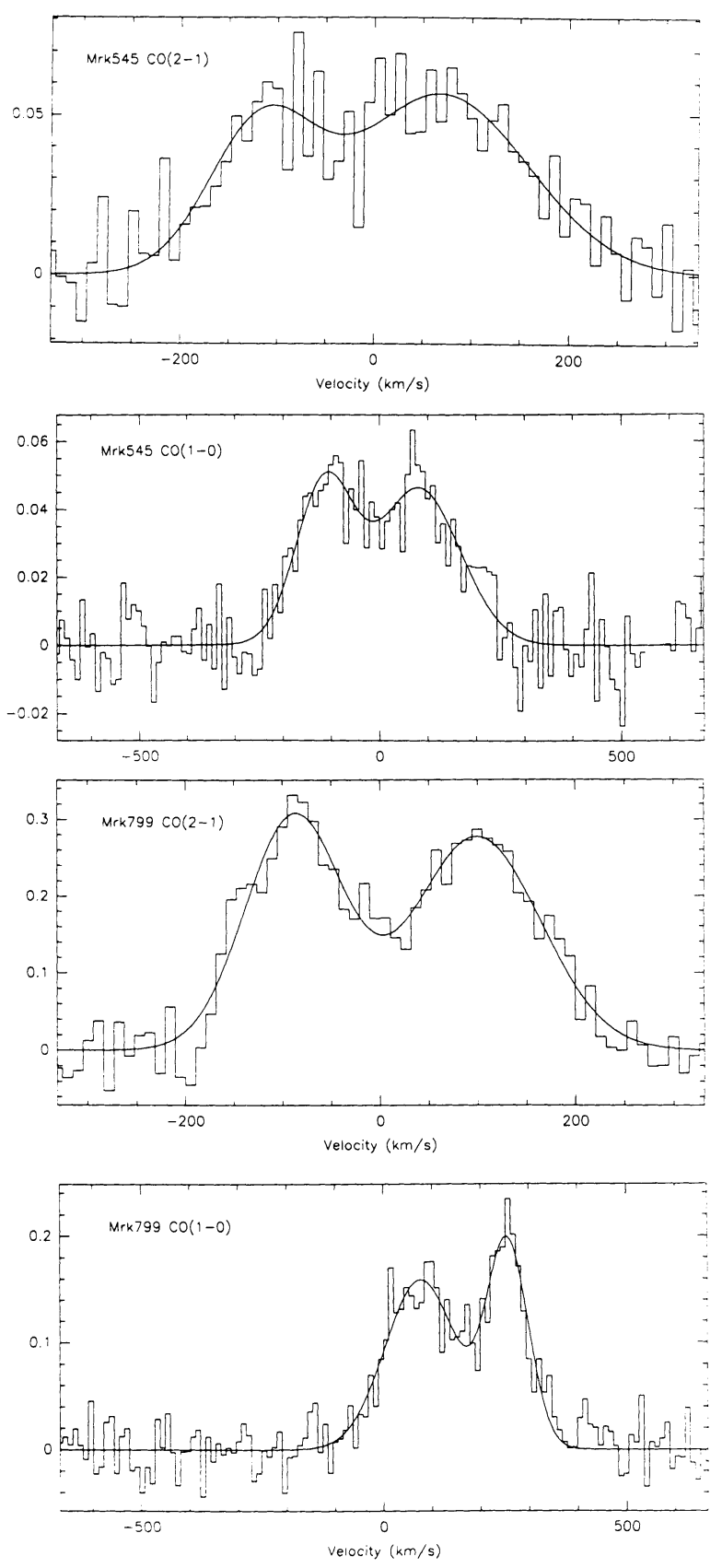